\title{
Very-high-energy GRB events in novel Fermi-LAT photon data and their emission mechanism
}

\author{
Mitsunari Takahashi* for the Fermi-LAT collaboration ${ }^{\dagger}$ \\ The Institute for Cosmic Ray Research, The University of Tokyo, Japan \\ E-mail: takhsmeicrr.u-tokyo.ac.jp
}

\begin{abstract}
The afterglow emission of gamma-ray bursts (GRBs) is basically well described by synchrotron radiation from fluid shocked by collisions between the jets and the external medium. However, several afterglow photons with energy from tens of $\mathrm{GeV}$ up to $94.1 \mathrm{GeV}$ have been detected by the Fermi Large Area Telescope. Such energy is challenging for the synchrotron radiation from the external shocks and may require another component such as inverse-Compton scattering.

Identifying the responsible emission process in this energy range is important for revealing the central engine and energy dissipation process which causes the bursts. In the energy range above $\sim 10 \mathrm{GeV}$, the sensitivity of the telescope is limited by the signal statistics. We developed novel photon classes of the Fermi Large Area Telescope to recover untapped events with energy above $20 \mathrm{GeV}$. Multivariate analysis for rejecting cosmic-ray backgrounds was optimized, and an increase of $\sim 70 \%$ in the signal acceptance around the peak energy, $\sim 100 \mathrm{GeV}$, was achieved.

In these classes, four candidates of photons correlated to GRBs were found. These events arrived much later than the end of the prompt phase. The observed energy of an event correlated to GRB $090926 \mathrm{~A}$ at $\sim 4.2 \times 10^{2} \mathrm{~s}$ after the trigger is $50 \mathrm{GeV}$. The redshift-corrected energy is $157 \mathrm{GeV}$. This is one of the highest energy values among GRB photon-like events detected with space telescopes. We estimated the coincidental background detection, and it turned out to be $(2.1 \pm 0.3) \times 10^{-4}$ counts. This energy exceeds the synchrotron energy limit for decelerated jets. In this case, another emission component such as inverse-Compton scattering is required.

These results demonstrated the possibility of the new data for scientific studies.
\end{abstract}

36th International Cosmic Ray Conference -ICRC2019-

July 24th - August 1st, 2019

Madison, WI, U.S.A.

\footnotetext{
* Speaker.

${ }^{\dagger}$ for collaboration list see PoS(ICRC2019)1177
} 


\section{Introduction}

Gamma-ray bursts (GRBs) are the most energetic explosions in the Universe. They are cosmologically distant, and each of them releases a huge amount of energy in keV-MeV gamma rays within a short period. Such "prompt" emission is followed by gradually decaying broadband "afterglow". The GRB emission is radiated by relativistic jets. Their progenitors, engines, mechanisms of jet acceleration and electromagnetic wave radiations are being debated. The afterglow emission is basically well explained by synchrotron radiation from fluid shocked by collisions between the jets and external medium. However, the Fermi Large Area Telescope (LAT) has detected several afterglow photons with energy from tens of $\mathrm{GeV}$ up to $94.1 \mathrm{GeV}$ in these ten years [1, 3, 2]. Above $50 \mathrm{GeV}$, three GRBs have been detected according to the second GRB catalog [2]. Such energy is too high for synchrotron radiation from the external shocks, especially in late time. It may require another component such as inverse-Compton scattering. Identifying the responsible emission process in this energy range is important for revealing the central engine and energy dissipation process which causes the bursts. For gamma rays with energy above $10 \mathrm{GeV}$, the sensitivity of the LAT is limited by the signal statistics in most cases. Then we have been trying to increase the number of the detectable gamma rays in the LAT data.

On 14th January 2019, Major Atmospheric Gamma Imaging Cherenkov (MAGIC) telescopes detected GRB 190114C with energy above $300 \mathrm{GeV}$ [4]. The observation started $\sim 50 \mathrm{~s}$ after the burst. To improve the LAT sensitivity above tens of $\mathrm{GeV}$ is important in order to cover both prompt and afterglow emission in the era of next-generation imaging atmospheric Cherenkov telescopes.

\section{Novel data classes of the Fermi Large Area Telescope}

\subsection{Large Area Telescope}

The LAT is an instrument for observing high-energy cosmic gamma rays with a wide field of view $(\mathrm{FoV})$ and unprecedented sensitivity for a space telescope. It is one of two instruments of the Fermi satellite, which was launched on 11 June 2008 [5] and normal science observation started on 4 August 2008 [6]. The LAT covers an energy range from $20 \mathrm{MeV}$ to higher than $300 \mathrm{GeV}$.

The LAT consists of three detectors, namely, the tracker (TKR), calorimeter (CAL), and the anticoincidence detector (ACD). When a high-energy gamma ray enters the LAT, it interacts with the materials and converts to an electron pair, and it produces an electromagnetic shower. The track and the deposited energy of the charged particles are measured mainly with the TKR and the CAL, respectively. The energy and incoming direction of the primary particle are reconstructed from this information. Veto signals from the ACD are used in order to reject cosmic-ray backgrounds.

\subsection{Calorimeter}

The CAL measures the amount and spatial distribution of the energy deposited by the shower. It is constructed of eight layers of twelve CsI crystal scintillator logs $(2.7 \times 2.0 \times 32.6 \mathrm{~cm})$. These layers are aligned alternately, and the logs have two photodiodes at each of the two edges. Each $\log$ is optically isolated. The position along each log where the scintillation light is emitted can be 
localized by evaluating the asymmetry of the signals at both ends. These two features enable us to reconstruct the $3 \mathrm{D}$ profiles of the showers. The total thickness of the CAL is 8.6 radiation lengths.

\subsection{Pass 8 analysis}

Pass 8 is the latest version of the LAT low-level analysis, which is periodically updated [7]. It was released on 24 June 2015 and replaced the former version Pass 7 for the entire mission data sets. The experience of observations in the early phase of the LAT mission let us know some unignorable features missed by the simulations. In Pass 8, new event reconstruction algorithms were introduced in order to prevent this effect from reducing the acceptance. Also, some updates in simulation, event reconstruction, and event selection realized a significant reduction in background contamination, an increase in the effective area, a better PSF, and more understood systematic uncertainties.

In Pass 8, multiple clusters of energy deposition are identified so that overlapping of the footprints of temporally proximate events are spatially distinguished [8]. This recovers 5-10\% effective area above $1 \mathrm{GeV}$ [7]. In the next step, momentum analysis is performed on every cluster identified in the previous step in order to determine the arrival direction of the primary particle. It reconstructs the event direction with an accuracy of a few degrees above $1 \mathrm{GeV}$.

\subsection{Calorimeter-only events}

The clustering analysis enabled us to develop new classes of untapped events. While the conventional event classes require information from the TKR for reconstructing events, the new event classes are meant to be used when there is no usable TKR information. Typically, such events pass through the TKR without interacting because its thickness is only $\sim 1.5$ radiation length, or enter the LAT from the side plains. They are dubbed "calorimeter-only (CalOnly)" events [9]. When the CAL direction is successfully reconstructed and one or more trajectories are found, the counterpart tracks are searched in the TKR. If no tracks agree to the CAL direction, it is categorized as a CalOnly event. The CalOnly and conventional standard classes are thus completely exclusive.

\subsection{Background rejection}

We have developed the background rejection and event classification of the CalOnly events and evaluated their performance with MC data. This is done for events above $20 \mathrm{GeV}$ because the shower axis can be determined by degrees and all events are recorded regardless of any other triggers. The measured signals of each event are parametrized, and they are used for the multivariate analysis (MVA) to discriminate gamma-ray signals from cosmic-ray backgrounds. The MVA method used for this study is the boosted decision trees. MC data sets for which the astronomical gamma rays and backgrounds are simulated are used for both training and evaluation. We optimized the MVA configuration for CalOnly events because the standard configuration has been constructed on the assumption that both TKR and CAL information are available, which does not happen for CalOnly events. We have evaluated the performance of MVAs with a variety of configurations in order to find the best one. In addition to selecting a good combination of parameters, we newly defined some helpful parameters. All of these steps are carried out by the Toolkit for Multivariate Data Analysis with ROOT [10]. 


\subsection{Event class definitions}

The standard gamma-like events are categorized into the nested event classes for scientific analysis. For the CalOnly events, we have defined four nested event classes with the gammalikeness evaluated by the MVA. The cut values of the gamma-likeness are determined based on the residual background rate relative to the level of the extragalactic gamma-ray background (EGB). The criteria are listed in Table 1.

Table 1: CalOnly event classes. A lower class is a sub-class of an upper class.

\begin{tabular}{|c||c|l|}
\hline Event class & Residual background & Uses \\
\hline \hline CalOnly_R100 & $\sim 10 \times$ EGB & Transients, timing studies \\
\cline { 1 - 2 } CalOnly_R030 & $\sim 3 \times$ EGB & \\
\hline CalOnly_R010 & $\sim 1 \times$ EGB & Point sources, moderately extended sources \\
\hline CalOnly_R003 & $\sim 0.3 \times$ EGB & Diffuse sources \\
\hline
\end{tabular}

\subsection{Performance}

The performance of the CalOnly classes is quantified as residual background rate, acceptance, point spread functions (PSFs), energy dispersion, and FoV. These characteristics are evaluated by MC simulation data of the signal gamma rays and background cosmic rays.

The acceptance is the effective area integrated over the FoV. It yields the signal statistics which we can get from a certain source during a long observation. It depends on the event energy $E$ and the off-axis angle from the telescope boresight $\theta$. The peak locates around $100 \mathrm{GeV}$. At the peak energy, the acceptance of the CalOnly_R100 and R003 corresponds to $\sim 70 \%$ and $\sim 40 \%$ of the standard SOURCE class, respectively. These fractions of photons are recovered for analysis on top of the conventional data. This is a significant improvement because, above $50-100 \mathrm{GeV}$, the LAT sensitivity is limited by statistics for most of the sources.

The PSFs strongly depend on $E$ and $\theta$. They are better for larger $\theta$ because the CAL is geometrically thicker and thus the tracks become longer. The $\theta$-integrated PSFs distribute from two to four degrees. These are more than one order of magnitude worse than those for the standard classes. The energy dispersion of the CalOnly events is comparable to that of the standard events although it also strongly depends on $E$ and $\theta$.

The CalOnly effective area is flatter for $\theta$ than the standard one, although the former is smaller than the latter at most of the angles. The relative gain of the CalOnly classes is larger at large $\theta$.

\subsection{Validation with flight data}

The developed classes must be validated by real data, not only by MC data. We produced an all-sky map with the CalOnly_R010 photons. The Galactic disk and some bright point sources such as Crab nebula and Mkn 421 are obvious. Next, we evaluated the consistency between the IRFs based on MC and the observed CalOnly data, using data of a bright gamma-ray point source, Mkn 
421. We analyzed standard data around Mkn 421 and constructed a spectrum model of gamma-ray sources with the result. Convolving the model and the CalOnly IRFs yields a prediction of the CalOnly data. We found that the model and the CalOnly data are roughly consistent in the event distribution of angular separation from the source.

\section{Calorimeter-only photon search}

We applied the developed data analysis for GRBs. The CalOnly classes improve the statistics of gamma rays with energy above $20 \mathrm{GeV}$, where synchrotron emission is difficult even from relativistic jets. We searched for CalOnly events spatially and temporally coincident with LAT-detected GRBs.

\subsection{GRB selection}

We selected the GRBs which were detected by the LAT by the end of $2016^{1}$ with one or more photons with energy above $10 \mathrm{GeV}$ and localized by $<0^{\circ} .3$. The information was taken from the online LAT GRB catalogue [11], the GBM catalogue [12, 13, 14], and the third Swift GRB catalogue [15]. Consequently, the number of GRBs which meet the criteria is 24. The highest photon energy is $94 \mathrm{GeV}$; the photon was coincident with GRB 130427A.

\subsection{Data selection}

All triggered events above $20 \mathrm{GeV}$ are recorded by the LAT. This enables us to revisit all archival GRB data in this energy range. We selected the CalOnly_R100 (the largest class) data of the 24 GRBs. The time window is from the GBM trigger time $T_{0}$ to $T_{0}+10 \mathrm{ks}$, where $T_{0}$ is the trigger time taken from the GBM catalogue. The region of interest (RoI) was configured as a circular region whose center was at the position of each GRB with the radius of (the PSF 68\% containment) + (the GRB localization error). The GRB position and its error were taken from the LAT online catalogue [11]. The PSF is a function of $E$ and $\theta$ of each event. It was determined with MC. We consider any event which meets all of these criteria as a candidate of a GRB photon. Hereafter the number of the CalOnly events found in the data of each GRB is denoted by $N_{O N}$.

\subsection{Background estimation}

The standard photon classes are almost background-free above $20 \mathrm{GeV}$ because both the temporal and the spatial information effectively suppress the backgrounds. However, the residual backgrounds are not negligible in the CalOnly classes because of their more than one order of magnitude worse PSFs. The residual background is composed of the residual cosmic rays, gamma rays from nearby sources, the EGB, the local diffuse gamma rays, and the Earth-limb gamma rays.

Since the background templates have not yet been implemented for the CalOnly classes, the standard likelihood analysis was unavailable. Hence, we performed ON/OFF analyses for estimating the probability that each CalOnly detection was caused by a coincidental background. The predicted count of the coincident backgrounds $N_{B K G}$ is calculated by Eq. 3.1.

$$
N_{B K G}=N_{O F F} \mathscr{E}_{O N} / \mathscr{E}_{O F F}
$$

\footnotetext{
${ }^{1}$ The search window will be extended to the latest data in the future.
} 
where $N_{O F F}$ is the observed count in the OFF data, $\mathscr{E}_{O N}$ and $\mathscr{E}_{O F F}$ are the exposure of the ON and OFF data, respectively. The raw background rate and the residual rate depend on $E, \theta$, and the zenith angle $\theta_{Z}$ besides the exposure $\mathscr{E}_{O N}$. The ON and OFF event distributions in the parameter space of $\theta_{Z}$ vs. $\theta$ vs. $E$ differ, and the difference was taken into account. As the OFF sample, we utilized the high-galactic-latitude regions defined in [6] for calibrating the LAT standard analysis. The data set is for 8.3 years and statistically large. This enables us to access the 3D dependence on ( $\theta_{Z}$ vs. $\theta$ vs. $E$ ). Once the predicted background is obtained, one can evaluate the false detection probability with the Poisson distribution.

\subsection{Detected calorimeter-only events}

Analyzing the data of the 24 GRBs recovered four gamma-like CalOnly events coincident with three GRBs: 090926A, 150902A, and 160509A. Their information and the background estimation results is reported in Table 2. In this contribution, we focus on an event related to GRB 090926A, which has a high gamma-likeness, good spatial agreement. The predicted background is $2.1 \pm$ $0.3) \times 10^{-4}$ events, and thus the false detection probability is $0.021 \%$. In Fig. 1, all CalOnly events around the GRB in the data for 8.3 years are shown. The observed and the redshift-corrected energy is $50 \mathrm{GeV}$ and $157 \mathrm{GeV}$, respectively.

Gamma-like CalOnly events around GRB 090926A for $8.3 \mathrm{yr}$

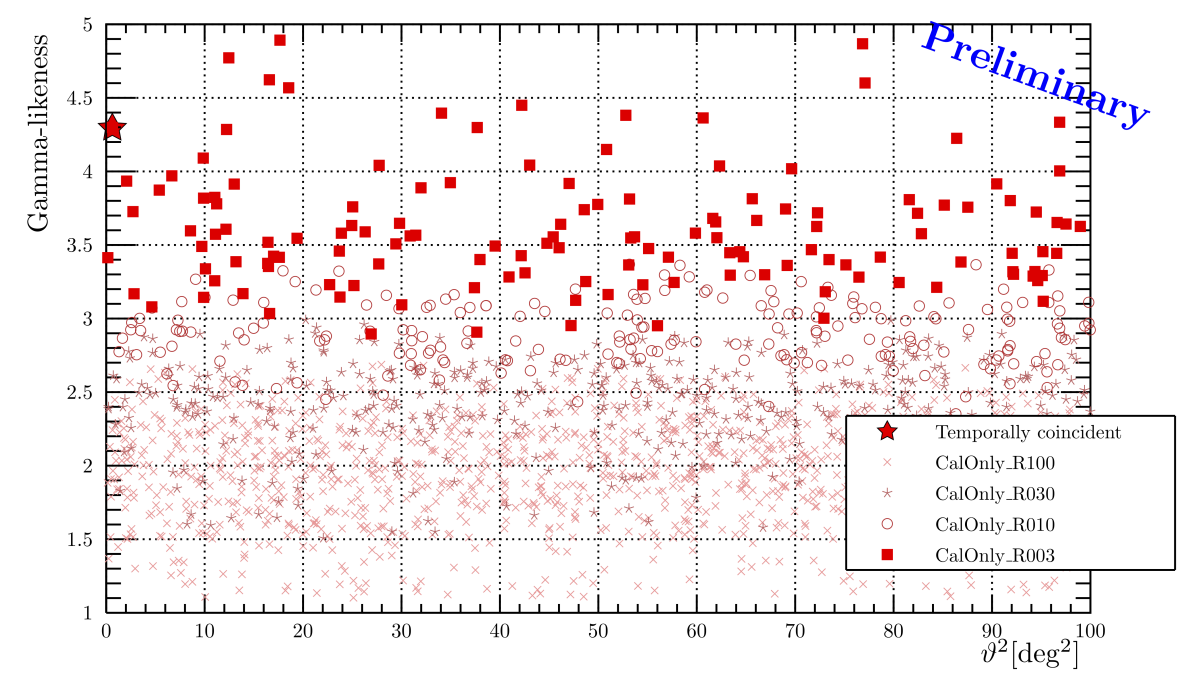

Figure 1: Gamma-likeness vs. $\vartheta^{2}$ of the CalOnly events for 8.3 eyars where $\vartheta$ is the angular separation of the event arrival direction of GRB 090926A [11]. The event which was temporally coincident to the GRB is highlighted by a star.

\section{Discussion and conclusions}

\subsection{Maximum synchrotron energy}

Synchrotron photons have possible maximum energy determined from the Larmor time [18]. It is $\approx 50 \mathrm{MeV}$ for electrons in their rest frame. Here we compare this to the energy of the CalOnly 
Table 2: Characteristics of the CalOnly events correlated with three of the 24 GRBs

\begin{tabular}{|l||r|r|r|r|}
\hline Coincidental GRB & 0909026A & 150902A & \multicolumn{2}{|c|}{$160509 \mathrm{~A}$} \\
\hline \hline Event class & CalOnly_R003 & CalOnly_R030 & CalOnly_R100 & CalOnly_R100 \\
\hline Arrival time & $\mathrm{T}_{0}+424 \mathrm{~s}$ & $\mathrm{~T}_{0}+2064 \mathrm{~s}$ & $\mathrm{~T}_{0}+2057 \mathrm{~s}$ & $\mathrm{~T}_{0}+5779 \mathrm{~s}$ \\
\hline Energy & $50 \mathrm{GeV}$ & $84 \mathrm{GeV}$ & $116 \mathrm{GeV}$ & $63 \mathrm{GeV}$ \\
\hline Angular separation & $0^{\circ} .8$ & $3^{\circ} .6$ & $4^{\circ} .6$ & $3^{\circ} .7$ \\
\hline cf. PSF68\% & $1^{\circ} .7$ & $5^{\circ} .2$ & $5^{\circ} .6$ & $5^{\circ} .0$ \\
\hline Off-axis angle & $62^{\circ}$ & $8^{\circ} .7$ & $9^{\circ} .9$ & $9^{\circ} .5$ \\
\hline Redshift & $2.1062[16]$ & unknown & $1.17[17]$ \\
\hline Energy in GRB-frame & $157 \mathrm{GeV}$ & $>84 \mathrm{GeV}$ & $252 \mathrm{GeV}$ & $137 \mathrm{GeV}$ \\
\hline Predicted background & $(2.1 \pm 0.3) \times 10^{-4}$ & $\cdots$ & \multicolumn{3}{|c|}{$0.063 \pm 0.005$} \\
\hline
\end{tabular}

event coincident with GRB 090926A. The observable energy is proportional to the Lorentz factor of the emission region $\Gamma$. Provided the density of the ambient matter is spatially constant, one can calculate the Lorentz factor of the shocked fluid with a weak dependence on the jet energy and the ambient matter density. In the case of GRB 090926A, $\Gamma$ is expected to be decelerated to $\lesssim 800$ at $t_{o b s}=\mathrm{T}_{0}+424 \mathrm{~s}$, the arrival time of the CalOnly event. Then, the synchrotron limit in the observer frame is $\lesssim 13 \mathrm{GeV}$. The CalOnly event energy $50 \mathrm{GeV}$ is about four times higher than it. This result suggests that another component such as inverse-Compton scattering exists on top of the synchrotron emission. An alternative possibility is that the energy injection to the external shocks continued much longer than the observed prompt emission by the GBM.

\subsection{Advantages of the new data classes}

The LAT has detected more than 140 GRBs since 2008 [11]. The large FoV has enabled us to access such a large number of bursts and observe many of them from their beginning. This feature is complementary to observations with imaging atmospheric Cherenkov telescopes. They have four orders of magnitude larger effective area although they start to observe tens of second after the burst. The CalOnly classes reinforce the advantages of the LAT observation. They increase the effective area in the signal-dominant regime and enlarge the FoV.

The angular resolution of the CalOnly events is poor, and it results in more background contamination in data of unextended sources compared with data with usable tracker information. However, the temporal information effectively suppresses the background contamination for transient objects and timing studies. Therefore, GRBs, AGN flares, and millisecond pulsars are good targets of the CalOnly classes. In addition, objects which do not require a good PSF could also benefit. One example is dark matter line searches at broad regions such as the Inner Galaxy. The event coincident with GRB 090926A demonstrated the possibility of the CalOnly classes. They will be publicly available as a part of the standard photon data in the future. 


\section{Acknowledgments}

The Fermi-LAT Collaboration acknowledges support for LAT development, operation and data analysis from NASA and DOE (United States), CEA/Irfu and IN2P3/CNRS (France), ASI and INFN (Italy), MEXT, KEK, and JAXA (Japan), and the K.A. Wallenberg Foundation, the Swedish Research Council and the National Space Board (Sweden). Science analysis support in the operations phase from INAF (Italy) and CNES (France) is also gratefully acknowledged. This work performed in part under DOE Contract DE-AC02-76SF00515.

\section{References}

[1] M. Ackermann et al., The First Fermi-LAT Gamma-Ray Burst Catalog, ApJS, 209, 11

[2] M. Ajello et al., A Decade of Gamma-Ray Bursts Observed by Fermi-LAT: The Second GRB Catalog, ApJS, 878, 1

[3] M. Ackermann et al., Fermi-LAT Observations of the Gamma-Ray Burst GRB 130427A, Science, 343, $42-47$

[4] R. Mirzoyan et al., MAGIC detects the GRB 190114C in the TeV energy domain, GCN CIRCULAR, 23701

[5] W. B. Atwood et al., The Large Area Telescope on the Fermi Gamma-Ray Space Telescope Mission, ApJ, 697, 1071

[6] M. Ackermann et al., The Fermi Large Area Telescope on Orbit: Event Classification, Instrument Response Functions, and Calibration, ApJS, 203, 4

[7] W. Atwood et al., Pass 8: Toward the Full Realization of the Fermi-LAT Scientific Potential in proceedings of 2012 Fermi Symposium, eConf C121028, [astro-ph.IM/1303.3514]

[8] P. Bruel, Gamma rays, electrons and positrons up to 3 TeV with the Fermi Gamma-ray Space Telescope, Journal of Physics: Conference Series, 404, 012033

[9] M. Takahashi et al., Calorimeter-only analysis of the Fermi Large Area Telescope in proceedings of 2014 Fermi Symposium, eConf C14102.1, [astro-ph.HE/1503.01364]

[10] A. Hoecker, TMVA - Toolkit for Multivariate Data Analysis, [physics/0703039]

[11] D. Kocevski et al., Fermi LAT GRBs, https://fermi.gsfc.nasa.gov/ssc/observations/types/grbs/lat_grbs

[12] D. Gruber et al., The Fermi GBM Gamma-Ray Burst Spectral Catalog: Four Years of Data, ApJS, 211, 27 [astro-ph.HE/1401.5069]

[13] A. von Kienlin, The Second Fermi GBM Gamma-Ray Burst Catalog: The First Four Years, ApJS, 211, 13, [astro-ph.HE/]

[14] P. Narayana Bhat, The Third Fermi GBM Gamma-Ray Burst Catalog: The First Six Years, ApJS, 223, 28, [astro-ph.HE/1603.07612]

[15] A. Lien et al., The Third Swift Burst Alert Telescope Gamma-Ray Burst Catalog, ApJ, 829, 7

[16] D. Malesani et al., GRB 090926A: VLT/X-shooter redshift, GCN CIRCULAR, 9942

[17] N. R. Tanvir et a al., GRB 160509A Gemini North redshift, GCN CIRCULAR, 19419

[18] O. C. de Jager, Gamma-Ray Observations of the Crab Nebula: A Study of the Synchro-Compton Spectrum, ApJ 457, 253 